recurrence at an average of 1.7 years after documented disease inactivity. Patients with generalized morphea experienced higher recurrence rates than those with linear morphea (HR: 3.03, 95\% Cl: I.48-6.22), and those treated with UVAI phototherapy had higher recurrence over those treated with methotrexate (HR: $2.33,95 \% \mathrm{Cl}$ : I.03-5.3I). In patients with follow-up periods longer than 5 years $(n=50)$, disease recurrence was observed in $44 \%$ of patients and the majority of recurrence represented new activity in an area of pre-existing morphea (82\%). DISCUSSION/SIGNIFICANCE OF IMPACT: This study highlights the previously under-studied dynamic long-term course of morphea, and identifies the clinical characteristics that predispose patients to having a relapsingremitting course. We conclude that patients with morphea should be followed closely over time even in the absence of disease activity due to the potential for disease recurrence.

\section{Mixed meal effects of neprilysin inhibition}

Jessica R. Wilson', Daniel Rader ${ }^{2}$, Raymond Townsend ${ }^{3}$ and Michael Rickels $s^{2}$

' University of Pennsylvania School of Medicine; ${ }^{2}$ Department of Medicine, Division of Nephrology and Hypertension, University of Pennsylvania; ${ }^{3}$ Department of Medicine, Division of Endocrinology, University of Pennsylvania

OBJECTIVES/SPECIFIC AIMS: Test the hypothesis that neprilysin inhibition with sacubitril/valsartan will increase endogenous intact GLP-I after a mixed meal compared with valsartan. METHODS/STUDY POPULATION: Adults I880 years with pre-diabetes or type 2 diabetes and elevated blood pressure. RESULTS/ANTICIPATED RESULTS: We anticipate higher intact GLP-I area under the cure after the meal when subjects receive sacubitril/valsartan compared with valsartan. DISCUSSION/SIGNIFICANCE OF IMPACT: Neprilysin inhibition may be a target for anti-diabetes therapy by decreasing degradation of GLP-I.

\section{3}

National dissemination of the accrual to clinical trials (ACT) network across the Clinical and Translational Science Award (CTSA) Consortium*

Elaine H. Morrato and Lindsay Lennox

University of Colorado at Denver

OBJECTIVES/SPECIFIC AIMS: The ACT Network was developed by 46 members of the CTSA Program hubs in collaboration with NCATS to help investigators explore and validate feasibility of clinical studies in real-time using linked electronic health record data for cohort discovery. ACT is being disseminated nationally across the CTSA consortium. METHODS/ STUDY POPULATION: Diffusion of Innovation Theory and Lean Start-Up principles inform dissemination strategies. Core materials were developed nationally and are being tailored to meet local CTSA dissemination norms. An advisory board, with expertise in communications, journalism, customer channel management, pharmaceutical commercialization and health IT entrepreneurship, is providing strategic advice to develop and refine dissemination strategies. Evaluation of dissemination methods will include network usage and web analytics for the ACT Network's interactive digital content and log-in portal, and surveys-interviews of ACT users using the RE-AIM implementation framework. RESULTS/ANTICIPATED RESULTS: Formative research identified ACT's primary value proposition for clinical researchers: "Explore patient populations in depth, in real time, from your desktop;" "Confirm study feasibility by iteratively testing and refining inclusion and exclusion criteria;" "Demonstrate feasibility in funding proposals and IRB submissions;" and "Identify collaborating sites for multisite studies by searching for patients across the CTSA network." Early dissemination metrics, including number-type of registered users, queries performed, and web analytics, will be presented. DISCUSSION/SIGNIFICANCE OF IMPACT: Researchers nationwide face common barriers in accruing enough participants for clinical trials. The inability to identify the right number and type of people to participate often makes clinical trials slow and costly. Better cohort discovery at the protocol development phase is a necessary requirement. By end of 2018, the ACT Network will

*Submitted on behalf of the ACT Network Research Team. reach $60 \%$ of the CTSA consortium providing a new tool for investigators to improve the design and execution of clinical trials.

Obstructive sleep apnea as an independent predictor of postoperative delirium and pain: An observational study of a surgical cohort

Patricia K. Strutz, Vanessa L. Kronzer, Mark D. Willingham, Amrita

Aranake-Chrisinger, William S. Tzeng, Arbi Ben Abdallah, Simon

Haroutounian and Michael S. Avidan

Institute of Clinical and Translational Sciences, Washington

University in St. Louis

OBJECTIVES/SPECIFIC AIMS: To study the role of OSA as an independent predictor of perioperative outcomes. METHODS/STUDY POPULATION: For this single-institution cohort study, we included data from patients who were enrolled into I of 3 prospective parent studies. All participants underwent inpatient surgeries, excluding neurosurgeries, which required general anesthesia and a postoperative stay of at least I day. Patients included in this study were assessed daily for postoperative delirium and pain severity as part of the parent studies. In the current study, determination of delirium diagnosis was based on the 3-minute Diagnostic Confusion Assessment Method (3D-CAM), and the Visual Analogue Pain Scale (VAS) was used for pain severity. Data on OSA diagnosis (determined by sleep study); OSA risk (determined by the STOP-Bang tool; snoring, tiredness, observed apnea, high blood pressure, body mass index $>35 \mathrm{~kg} / \mathrm{m}^{2}$, age $>50$, neck circumference, male gender); and compliance with treatment were obtained from the preoperative assessment record. Participants were grouped into I of 3 categories: high risk of OSA (HR-OSA including patients with a previous positive sleep study or STOP-Bang score $\geq 5$ ) intermediate risk of OSA (IR-OSA; including patients with a STOP-Bang score of 3 or 4); and low risk of OSA (LR-OSA; including patients with a previous negative sleep study or STOP-Bang score $<3$ ). Candidate risk factors for delirium and pain were also extracted from this record. RESULTS/ANTICIPATED RESULTS: Logistic regression will be used to test whether OSA independently predicts postoperative delirium and linear regression to assess OSAs relationship to acute pain severity. We hypothesize that patients in the HR-OSA category will experience a higher incidence of postoperative delirium and greater postoperative pain severity. We also predict a step-wise increase in risk of these adverse outcomes when analyzing patients stratified by OSA risk (HR-OSA vs. IR-OSA vs. LR-OSA). For our secondary analyses, we anticipate these outcomes are modified by compliance with CPAP treatment. We believe patients with OSA who do not use prescribed CPAP will experience a higher incidence of postoperative delirium as well as increased pain severity. DISCUSSION/SIGNIFICANCE OF IMPACT: OSA is a common and frequently undiagnosed perioperative problem associated with altered pain processing and a high incidence of postoperative delirium. While likely providing stronger evidence of OSA's reported impact on postoperative delirium and pain, our findings might also help discern points of intervention for treatment and prevention. Since OSA's presumed impact poses challenges to clinicians and patients, prospective, randomized trials testing preventative or mitigating interventions are necessary. We hope to use these results to design such trials and clinical plans, with the goal of reducing postoperative delirium and acute postsurgical pain severity for the large number of patients at risk due to OSA.

Pembrolizumab for patients with leptomeningeal disease from advanced solid tumors Jarushka Naidoo, Chen Hu, Karisa Schrek, Roisin Connolly, Cesar Santa-Maria, Evan Lipson, Ranee Mehra, Bettegowda, Kristin Redmond, Arun Venkatesan and Stuart Grossman Johns Hopkins University School of Medicine

OBJECTIVES/SPECIFIC AIMS: Pembrolizumab is an anti-PD-I immune checkpoint antibody that has demonstrated promising anti-tumor activity in patients with solid tumor malignancies, including patients with brain metastases from malignant melanoma and non-small cell lung cancer. Leptomeningeal disease (LMD) is a rare form of malignant spread to the central nervous system (CNS), that occurs in $2 \%-10 \%$ of patients with solid tumors, most commonly in breast cancer and non-small cell lung cancer. We propose an open-label phase ll study of pembrolizumab in patients with LMD from advanced solid tumors 